\title{
Spatially-resolved volume monitoring of adhesive cure using correlated-image optical coherence
}

\section{tomography}

\author{
H. D. Ford* and R. P. Tatam
}

Department of Engineering Photonics, School of Engineering, Cranfield University, Cranfield, Bedford, MK43 0AL, UK.

${ }^{*}$ Corresponding author.

Tel. +44 (0)1234 750111, Fax +44 (0)1234 752452, e-mail h.d.ford@cranfield.ac.uk

Keywords: non-destructive testing, cure/hardening, optical coherence tomography.

\begin{abstract}
Cross-correlation of optical coherence tomography $\left(\mathrm{OCT}^{1}\right)$ images has been applied to internal imaging of PVA and two-part epoxy adhesives during cure, providing information on relative viscosity at different positions within the sample volume. Spatial resolution of a few micrometres is obtained in the original OCT images, and a few tens of micrometres in the correlation images, enabling the progress of cure to be mapped in fine detail within a small cure volume. Evidence of phase separation is seen in the OCT and correlation images of a partially-cured PVA emulsion. Mixing structure and regions of poor cure can be observed in a poorly-mixed two-part epoxy.
\end{abstract}

\section{Introduction}

Monitoring the progress of cure in an adhesive is of interest to enable changes in behaviour to be studied when one or more of the factors affecting cure are varied [1]. These might include: ambient conditions such as temperature, humidity or lighting; variability between production batches, variation of the thickness or volume of material to be cured, or alteration of the chemical composition due to aging of the uncured adhesive. A detailed and accurate knowledge of the cure process minimises the time that must be allowed before the cured part can be used or further processed, and enhances understanding of the physical and chemical processes taking place as cure progresses [2].

\footnotetext{
${ }^{1}$ OCT - Optical coherence tomography
} 
A range of cure-monitoring techniques exists. For exothermic reactions, it is possible to track progress via measurement of the temperature at one or more positions within the cure region. This can be achieved either remotely using, for example, an infra-red detector array [3], or via a contact technique involving thermo-chromic materials or insertion of miniature temperature probes into the liquid adhesive [4].

Other methods are required that are more widely applicable. The available techniques follow changes in either the mechanical or electrical properties of the adhesive as cure progresses. Acoustic sensors are commonly used, relying on one of two principles: in the first type of sensor, the resonant frequency of the sample, which evolves during cure, is monitored continually following excitation with broadband, low-frequency sound [5]. In the other technique, ultrasonic sound pulses are coupled into the cure zone via a contact transducer, allowing the changing velocity of sound to be monitored as the material modulus evolves during cure [6].

Dielectric measurement methods are the most sophisticated of all the cure-monitoring techniques, and provide the most detailed information; an alternating voltage is applied across the cure region, using contact electrodes positioned at two or more sites on the adhesive, and resulting in an induced current within the material. By monitoring the electrical parameters, including voltage, current, the relative phase between the two and the decay of electrode polarisation upon removal of the excitation signal, properties of the adhesive mix can be studied over time [7].

Optical monitoring techniques include fluorescence methods [8] and near-infra-red spectrometry [9]. Optical-fibre techniques are also used on occasion. Of these, the most widely known makes use of one or more optical-fibre Bragg gratings embedded into the adhesive cure zone [10]. A Bragg grating comprises a refractive-index modulation, written into the fibre core, which acts as a mirror for one very narrow optical wavelength band. Broadband light is coupled into the fibre and the Bragg reflection is monitored. The reflection wavelength is sensitive to the precise grating spacing, which is both strain and temperature dependent. In a non-exothermic reaction, the strain experienced by the 
optical fibre indicates the progress of cure [11]. Strain and temperature can, in some circumstances, be monitored simultaneously [12].

Other optical-fibre devices, such as tilted or long-period gratings, allow light to be coupled out of the fibre core into the surrounding cladding glass. Here they experience an "effective refractive index" modified by the refractive index value of the surrounding medium. Subsequent re-coupling of the light into the fibre core, by a second grating, allows the modified index to be measured, providing another method of following the cure process [13].

\subsection{Optical coherence tomography}

A feature common to the techniques outlined above is that they produce a spatially integrated output signal. In other words, the value of the measurand, at any point in time, corresponds to an average either over the entire cure region or over an extended zone within that region.

Optical coherence tomography (OCT) is an optical imaging methodology that uses low-power radiation in the near infra-red region of the spectrum [14]. It uses low-coherence interferometry to provide structural images of turbid biological tissue, with a spatial resolution of a few micrometres, to a depth of 1-2 mm below the surface, and has developed rapidly over the past two decades into a valuable tool for medical applications, particularly in diagnosing early-stage cancers and ophthalmological diseases $[15,16]$.

We propose developing and applying a recently-published, image-correlation OCT technique [17] to create a useful addition to the suite of methods available for adhesive cure monitoring. The advantage of the proposed technique is its ability to pinpoint, with very high spatial resolution, the locus of a particular degree of cure within a sample area having dimensions of the order of one millimetre. This permits, in effect, a time-series of images to be produced depicting the movement of a "cure front" within a small volume of adhesive; something that is not possible using any of the currently-available cure-monitoring techniques.

Most commonly, OCT is used in a manner analogous to tomographic ultrasound, to provide a live display of sub-surface structural images with a resolution close to that achieved by histological 
microscopy [18]. Using a fast data acquisition system, images for post-processing can be acquired at rates from several Hertz up to many tens of Hertz, dependent on the number of pixels required in the image. For live imaging, on-line processing and display implies a somewhat lower update rate. Very recently, a new OCT processing method was demonstrated, that involves the cross-correlation of successive images to identify regions of movement within the OCT image [17]. A similar technique has been used in the past, in which sub-surface velocity in diffuse objects is encoded as a contrast reduction in speckle images [19], but only now has OCT enabled the extension to depth discrimination within the images. Enfield et al [17] used the technique to differentiate regions of free fluid from regions of solid tissue in the living human arm, revealing a detailed map of the microvasculature. Their interest was purely in identifying the positions of the blood vessels, and they did not attempt to measure the velocity of flow in these experiments. However, it is clear that altering the time interval allowed between successive image acquisitions will affect the degree of correlation, as the positions of particles moving more rapidly within the sample will de-correlate faster than those of slow-moving particles. Where there is no pressure gradient within the fluid, the physical quantity detected by the OCT measurement is the displacement of scattering particles caused by Brownian motion, which is, in turn, related to the local viscosity. This is discussed at greater length in section 2.1.

\section{Theory}

The speckle-pattern changes caused by movement in the sample between acquisition of two sequential OCT images $A$ and $B$, is obtained by performing a cross-correlation of the two images [17]. This consists, in essence, of sub-dividing the images into small, square or rectangular sub-regions of pixel dimensions $M \mathrm{x} N$ and, for each pair of sub-regions, summing the products of intensities for all corresponding pixels in that pair, yielding a local correlation coefficient. A normalisation procedure is generally carried out at the same time to ensure that the final values obtained fall within a standard range, to allow for comparison of different experiments.

Mathematically, the cross-correlation for a particular sub-region at position $(x, y)$ in images $A$ and $B$, $C C_{A B}$, is given by 


$$
C C_{A B}=\sum_{p=0}^{M} \sum_{q=0}^{N}\left[\frac{\left(I_{A}(x+p, y+q)-\overline{I_{A}}\right)\left(I_{B}(x+p, y+q)-\overline{I_{B}}\right)}{\sigma_{A} \sigma_{B}}\right],
$$

where the mean intensity of the sub-region in $A, I_{A}$, is given by $\overline{I_{A}}=\left(\sum_{p=0}^{M} \sum_{q=0}^{N} I_{A}(p, q)\right) / M N$, the

standard deviation of the intensity, $\sigma_{A}$, in region $A$ by $\sigma_{A}=\sqrt{\sum_{p=0}^{M} \sum_{q=0}^{N}\left(I_{A}(x+p, y+q)-\overline{I_{A}}\right)^{2}}$, and similarly for the equivalent quantities in the same sub-region of image $B$.

It can be seen from equation 1 that, if the pixel intensities for this sub-region are identical in images $A$ and $B$, both the numerator and the denominator are equal to the (identical) variance of either image, and we obtain the maximum possible value for the correlation coefficient, $C C_{A B}=1$. Conversely, if the relative values of each pixel in the paired sub-regions are equal in magnitude but opposite in sign, we obtain the lowest possible value $C C_{A B}=-1$.

For the OCT images used in this project, spatial and velocity scales are similar in both the $x$ and $y$ axes and it is convenient to use square sub-regions with $M=N$. To avoid excessive loss of resolution in the correlated images, the size of the sub-region, also known as the kernel, should be selected such that both the OCT image intensity and the particle velocity can be considered approximately constant within the kernel, for the majority of kernels across the image. Very large kernels result in smearing of the data and take a long time to process, whereas very small kernels can introduce excessive noise into the correlation images. Kernel size has been selected empirically for results presented below, with sizes of between $5 \times 5$ to $7 \times 7$ pixels found to offer a good compromise between noise levels, processing time and retention of spatial detail.

Prior to the above procedure, global cross-correlations were performed to identify any bulk motion of the image, with a kernel size equal to the entire image and calculating the correlation coefficient for a small range of positive and negative pixel shifts in $x$ and $y$. Having identified the shift values resulting in optimum overall correlation, the main cross-correlation calculations were performed using the same 
offsets. However, it was found in these experiments that in no case did the required offset in either axis exceed one pixel.

Anomalous correlation is obtained in regions of very low intensity due to a constant, non-zero background, relative to which any pixel intensity fluctuations are very small. A threshold is therefore set, at a level which excludes parts of the original images where structural data is obscured by noise and constant background intensity.

\subsection{Scattering and Brownian motion}

The possible causes of variation in optical scattering during adhesive cure must be considered.

Scattering can occur in the presence of density and refractive-index fluctuations, on the same scale as the wavelength of the incident light, which arise as chemical and physical processes take place during cure [20]. However, where refractive-index variations are small, this scattering is generally weak compared with that from particles in the fluid and, since only a low-intensity OCT signal is obtained from optically-clear epoxies, we have inferred that the effect can be neglected in the current work. Following an initial settling period after sample deposition, during which some bulk flow of the fluid is likely to occur, it is assumed that Brownian movement of particles within the adhesive mix is the dominant cause of speckle movement. Generally, the scattering measurements are aided by the filler particles contained in many commercial adhesives, which help to provide a strong OCT signal. Where these are absent, it would in many cases be possible to seed the mix with particles, specifically to provide the required scattering signal, though it must be borne in mind that this could also alter other properties of the mix.

Brownian motion refers to the motion of particles suspended in a fluid. Such particles move in a random fashion as they experience collisions from all directions with the smaller liquid molecules that surround them [21]. Particles undergoing Brownian motion obey the diffusion equation which, in its one-dimensional form, states that the density $\rho(x, t)$ of particles at a point $x$ and time $t$ is given by $\partial \rho / \partial t=D\left(\partial^{2} \rho / \partial x^{2}\right)$, where $D$ is the mass diffusivity [22]. The solution of this equation leads to the result $D=\overline{x^{2}} / 2 t$, which implies that the distance travelled by the particle is proportional to the square 
root of the time interval. The diffusivity can be related to the viscosity of the fluid via the EinsteinSmoluchowski relation and the Stokes mobility formula, resulting in the expression $D=\left(k_{B} T\right) /(6 \pi \eta r)$, where $k_{B}$ is Boltzmann's constant, $T$ is temperature, $\eta$ is the viscosity and $r$ is the radius of the particle (assumed spherical). Hence the movement of a particle over time relates directly to the viscosity under a known set of ambient conditions.

In an adhesive cure application, additional interpretation of the OCT correlation images is required to obtain quantitative information, since the density of suspended particles is typically high and individual particles are not seen in the OCT images. The cross-correlation value obtained at a particular point is therefore dependent upon parameters including the integration time for each image point, and the optical speckle statistics, though the rate of change of speckle intensity will clearly be of the same order as the rate of change of particle position. Thus it is not generally straightforward to obtain an absolute, quantitative link between the correlation image and the physical properties of the adhesive. Nevertheless, much useful information is obtained: the time taken to achieve the same degree of cure at different points within the adhesive volume is ascertained, and cavities or inhomogeneities within the partially or fully cured fluid will sometimes be visible. Additional information is also available from the individual OCT images, including the physical shape of the fluid volume during and after cure, and the temporal variation of the average refractive index.

\section{Experimental methods}

A commercial OCT system (Thorlabs OCP930SR) at $930 \mathrm{~nm}$ was used to acquire the images used in these experiments. Instruments typically use wavelengths in the range $800-1550 \mathrm{~nm}$, and imaging in OCT is often improved at longer wavelengths because scattering is reduced. However, for samples containing a high proportion of water, absorption increases rapidly with wavelength in the near infrared, resulting in significant optical power reduction at the longer NIR wavelengths for depths greater than about 1-2 mm, outweighing the advantage from reduced scattering. In this study, the advantage was apparent only in the deeper regions of PVA glue samples. For aqueous media with lower 
scattering, where imaging might be performed to greater depths, absorption at wavelengths above $1400 \mathrm{~nm}$ could be severely limiting.

The Thorlabs OCT system is of the Fourier-domain (FD-OCT) type also known as spectral radar [23]. Light from a broadband SLD source is transmitted, via an optical fibre, to a small, portable head about the size of a mobile phone. Lenses and a galvanometer scanner in the probe head focus the beam to a spot diameter of about $8 \mu \mathrm{m}$, and sweep it across the surface of the sample, allowing information to be obtained within a region up to $8 \mathrm{~mm}$ long parallel to the sample surface and up to 1.6 millimetres deep, dependent on the turbidity of the sample. The thickness of the image plane, and the best lateral spatial resolution within the image, are about $8 \mu \mathrm{m}$. Depth resolution depends on the optical source bandwidth, and is specified as $6.5 \mu \mathrm{m}$ (in air) for this system. The returned OCT signal is processed using a spectrometer comprised of a grating and a 1024-pixel line-scan camera, the output of which is attached to a PC. Using this instrument, the minimum time interval between image acquisitions was fixed, depending on the precise image parameters, at a value of about 0.1-0.3 s, but longer time intervals were accessed by taking a series of images and using only those acquired at selected multiples of the base value in the correlation calculation.

Two time intervals are relevant in the acquisition of correlation images. Sets of three to six images were acquired at fixed times throughout the cure period. The time difference between acquisition of successive image sets is referred to as the 'sampling interval' images within each set being separated by appropriate 'correlation intervals' $\tau_{C}$, related to the viscosity of the sample and the size of the scattering particles. The sampling interval $\tau_{s}$ between each set of images was generally much greater, and was chosen to adequately sample the entire cure process, or the portion under study, at the largest rate of change encountered. Initially, test runs were performed on each sample to gauge the approximate time to full cure, subsequently enabling sensible sampling intervals to be chosen during various stages of the process.

To demonstrate the principle of correlation OCT for adhesive cure-monitoring, and to illustrate some of the additional information that can be obtained, a set of experiments was performed using two types 
of adhesive. A PVA, aqueous emulsion paper glue, of the type sold for crafts, was studied, as well as a two-part 'Araldite Rapid' epoxy. For the two-part epoxy resin, experiments were performed both on a thoroughly-mixed sample and a poorly-mixed sample, to look for differences between the two in correlation images obtained during and after curing. In all cases, image acquisition commenced within a minute or so of depositing the sample on the substrate; this time being required for horizontal and vertical positioning of the sample under the OCT imaging head.

The maximum optical depth for imaging, using the Thorlabs OCT system, is fundamentally limited by the number of pixels in the linescan camera of the spectrometer, since this determines the maximum interferometer fringe frequency that can be detected by the spectrometer, according to the Nyquist limit. For the Thorlabs system, this depth is $1.6 \mathrm{~mm}$ in air, and lower in dense materials, since the optical depth is defined as the product of the physical depth and the refractive index. The consequence is that beads or layers of adhesive, no more than about $1 \mathrm{~mm}$ in depth, are optimum for study using this system. Imaging is often limited to somewhat shallower depths due to optical power loss caused by strong scattering from the sample. A matt black substrate is desirable, as the OCT technique is extremely sensitive. Over-bright scattering from reflective surfaces can result in saturation of the detector and the introduction of image artefacts.

\section{Results and discussion}

\subsection{PVA}

In figure 1, original OCT images, acquired at various times following bead deposition, ranging from 1 minute to 1 day, are shown for a bead of PVA glue, about $0.8 \mathrm{~mm}$ in height, deposited on a blackanodised aluminium plate. All measurements were made at room temperature. The top surface of the bead appears uppermost in the OCT images. The surface of the aluminium plate, which can be identified as a bright horizontal line towards the sides of the images, cannot always be seen in the region below the glue bead. Initially, optical penetration is too low to reach the plate through the centre of the bead. However, as drying occurs, more of the substrate plate gradually becomes visible, and, in fact, appears curved in the region below the glue bead. The curvature is because, as stated 


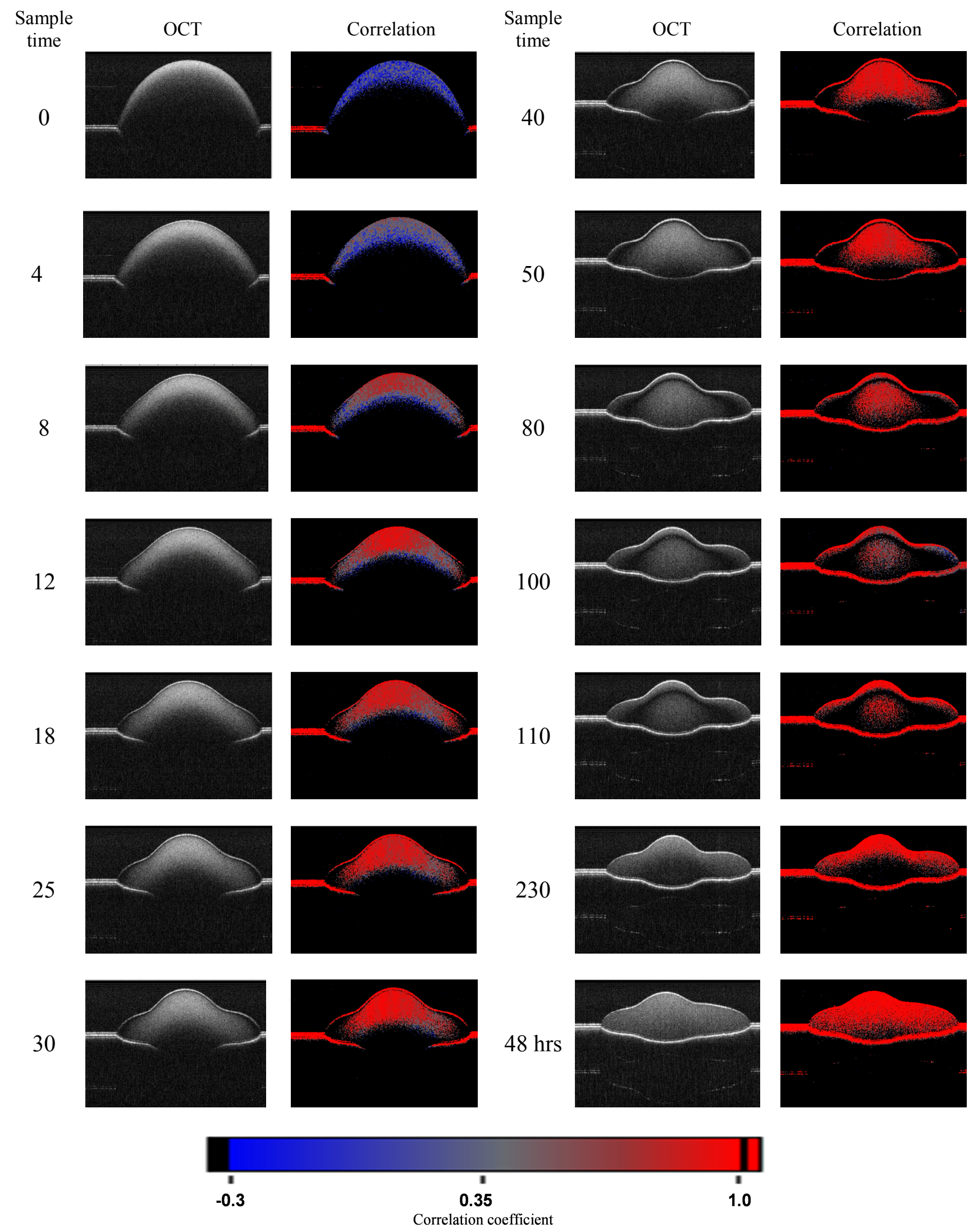

Figure 1. OCT (left) and correlation (right) images for the PVA paper glue sample. Image dimensions are 3 mm laterally by $1.6 \mathrm{~mm}$ deep. The sampling time given for each image pair is in minutes, except for the final pair, and the correlation interval is $15 \mathrm{~s}$ in all cases. 
above, the system measures optical depth, rather than physical depth. Thus the optical distance to the plate is greater, when viewed through a large depth of glue with a refractive index of around 1.3-1.5, than when viewed through the same distance of air, with a refractive index of 1.0.

Intensity scales of raw OCT images were constant within a data set. The height of the substrate was adjusted between image sets, to keep the top of the sample at approximately the same distance from the top of the image frame and maximise sensitivity, which drops off with depth. Therefore, intensities referenced to a common height relative to the substrate cannot be compared directly between images. Nevertheless, the increase in substrate brightness between images at 40 and 80 minutes is sufficiently high to be clearly indicative of an increase in sample transparency with time.

For the PVA glue, the scattering mechanism is somewhat different from that seen in semi-translucent epoxies. PVA paper glue is an aqueous emulsion, with tiny droplets of PVA acting as the scattering centres, hence the opaque appearance of the uncured adhesive. Additional filler particles are probably present as well, since the glue does not dry completely clear. Cure involves the evaporation of the water and coalescence of the PVA droplets.

Care should be exercised with the interpretation of results in images of this type of adhesive, because emulsion droplet size will change as the water content decreases, resulting in an alteration in the Brownian motion parameters. Because the PVA emulsion scatters strongly, the penetration depth is limited initially to less than the full depth of the bead. There is a loss of information at optical depths greater than about 300 micrometres, therefore the substrate plate is not imaged in the region towards the centre of the bead. However, as the glue loses water through evaporation, two effects operate: the physical dimensions of the bead start to shrink, and the scattering becomes slightly weaker, possibly due to agglomeration of PVA emulsion droplets. The combined effect is to allow deeper imaging. By the time an almost full cure is achieved, as shown in the final image of figure 1, the substrate can be seen through the entire volume of the bead, which now has a maximum depth of about 600 micrometres. 
Slight 'ghosting' that appears below the main OCT image in images at longer sample times is an artefact due to over-strong reflections from the substrate and the upper surface of the bead, and can be ignored.

\begin{tabular}{|c|c|c|c|}
\hline & Sample time range/mins & $\begin{array}{l}\text { Sampling interval } \\
\tau_{\mathrm{s}} / \text { minutes }\end{array}$ & $\begin{array}{l}\text { Correlation intervals } \\
\tau_{\mathrm{c}} / \text { seconds }\end{array}$ \\
\hline \multirow[t]{6}{*}{ PVA glue } & $0-20$ & 2 & $5,10,15$ \\
\hline & $20-35$ & 5 & $15,30,45$ \\
\hline & $35-50$ & 5 & $15,30,45,75$ \\
\hline & $50-70$ & 10 & $15,30,45,75$ \\
\hline & $70-110$ & 107 & \\
\hline & Then 240 mins, 1 day & & $15,30,60,90,120$ \\
\hline \multirow{5}{*}{$\begin{array}{l}\text { 'Araldite Rapid' } \\
\text { (Well-mixed and } \\
\text { poorly-mixed) }\end{array}$} & & 1 & \\
\hline & $\begin{array}{c}8-8 \\
8-8\end{array}$ & 2 & $10,20,40$ \\
\hline & $\begin{array}{c}8-20 \\
20-40\end{array}$ & 5 & $10,20,40,80$ \\
\hline & $\begin{array}{l}20-40 \\
40-60\end{array}$ & 10 & \\
\hline & Then 80,120 mins, 3 days & & $10,20,40,80$ \\
\hline
\end{tabular}

Table 1. Values for OCT sampling intervals (between image sets) $\tau_{s}$ and correlation intervals (between images within sets) $\tau_{c}$ used at different stages during the cure-monitoring of PVA and epoxy adhesive samples.

Following preliminary trial runs, a set of data was acquired for initial correlation intervals $\tau_{\mathrm{c}}$ of 5,10 and $15 \mathrm{~s}$ and a sampling interval of 2 minutes. Images were saved as text files, and the image correlation was carried out in post processing, using LabView software. As setting progressed, the rate of change of cure slowed, and larger values became appropriate for both the correlation interval $\tau_{\mathrm{c}}$ and the sampling interval $\tau_{\mathrm{s}}$. The values used during different stages of cure, for this experiment and subsequent experiments on epoxy samples, are displayed in table 1.

Space is insufficient in this paper to display image sets for all the correlation times listed in table 1; a single, representative correlation image at an appropriate value of $\tau_{\mathrm{c}}$ has therefore been selected for each sampling point. False-colour scaling has been applied to the correlation images, such that correlation coefficients above about 0.4 appear as light grey (red online), with correlation coefficients less than about 0.3 appearing dark grey (blue online). Intermediate values appear in mid grey. The most appropriate scaling will change under different imaging conditions and for different sample 
materials. Correlation images are shown next to the original OCT images, for the equivalent points in time.

Some interesting features are seen, both in the raw images and in the correlation data. It is clear that a skin of partially-cured material forms rapidly where the surface of the glue is exposed to the air, following which the cure process evolves inwards towards the centre of the bead. Assuming the initial composition of the bead to be homogeneous, pixels having equal values of correlation coefficient represent positions of equal viscosity. We must be more cautious in assuming that this is the same viscosity value in later images, because the liquid composition changes as the glue bead dries, with possible coalescence of emulsion droplets.

At the edges of the bead, where liquid forms a shallow contact angle with the substrate, a region of very low scattering appears within the first few minutes between the surface skin and the substrate material, resulting in an almost total loss of OCT information. This zone, which shows up as a dark region in the images, is strongly indicative of a phase change in the mixture. As cure proceeds, the dark zone slowly grows in width and moves inwards towards the centre of the bead, eventually disappearing in the fully-cured adhesive. As the dark zone moves inwards, it is followed by the reappearance of speckle in the outer regions of the bead. We note that the time constant for the speckle that re-forms outside the dark zone, as it migrates, is lower than that observed at a much earlier stage in the original mix. This implies the presence of three distinct phases within the bead of PVA, and the lack of scattering in the phase represented by the dark zone is indicative of a single component here. Initially, as evaporation takes place, a thickening of the emulsion occurs, with a concomitant increase in viscosity. It may then be that, as the fraction of water in the water/PVA emulsion drops to a certain concentration, a separation of the emulsion occurs, with the PVA component migrating towards the partially-cured outer region, and bordering a pure aqueous zone on the inner side. Further investigation is required to understand the process more fully.

\section{2 'Araldite Rapid' epoxy: well-mixed sample}






Figure 2. OCT (left) and correlation (right) images for a well-mixed 'Araldite Rapid' epoxy sample. Image

dimensions are $3 \mathrm{~mm}$ laterally by $1.6 \mathrm{~mm}$ deep. The sampling time given for each image pair is in minutes and the correlation interval is $40 \mathrm{~s}$ in all cases. Colour scale is as for figure 1.

Images are presented in figure 2 for a two-part 'Araldite Rapid' epoxy, mixed using approximately equal quantities of resin and hardener as directed on the packaging, with both original OCT data and the correlation images displayed for a selection of sample times. Again, scattering is quite high, limiting optical penetration, and high-quality data is obtained from only the upper two-thirds of the adhesive volume. In this experiment, the 'initial' cure time quoted for the mixed adhesive was about 10 minutes, while the quoted time to full cure was of the order of one hour. Because the original 
mixture was quite viscous, relatively long correlation intervals, given in table 1, were selected, with yet higher values used, in addition, as cure progressed. A short line of adhesive, about $1 \mathrm{~mm}$ in thickness, was set down on the anodised aluminium substrate, and image acquisition was started within 1 minute as before. Initially, very low correlation coefficients were observed for a correlation time constant $\tau_{C}$ of $30 \mathrm{~s}$. However, after about 6-7 minutes, the viscosity increased sufficiently that a $\tau_{C}$ of several minutes was required to obtain the same correlation coefficient values. The behaviour of this epoxy during cure is very different from that of the PVA glue. During about the first five minutes from mixing, there are no changes that produce a significant difference in the correlation images over the range of correlation times used here. This can be seen from the persistence of darker grey (blue online) colouration in the images over this period. During the following four or five minutes, however, a rapid increase in viscosity occurs throughout the imaged area, marked by an increase of light grey (red online) colouration in the images, corresponding to a high correlation coefficient. During this stage, the viscosity of the mixture becomes so high that stirring is no longer possible. The mix then enters a stage of continuing, slower cure, in which the correlation coefficient rises at a lower rate, eventually reaching a high fixed value, close to 1 , from which it does not change.

Some interesting features are seen in the images of figure 2. Bubbles appear, particularly in the early images, when viscosity is still relatively low, as dark vertical streaks of differing widths. Specular reflections from the smooth surfaces can be seen only near the centre-line of the bubble where the surface normal is close to the optical axis. Total internal reflection will occur over roughly the outer half of the upper hemisphere of the bubble. The probe beam, and scattered light returning upwards from below the bubble, will be distorted by refraction at the curved surfaces, resulting in defocus effects that account for the pronounced shadowing. Large opaque inclusions can cause similar occlusion of lower regions of the mix, whereas smaller inclusions appear in some images as particularly bright scattering centres. Figure 3 shows an expanded view of an OCT image containing both bubbles and a highly-scattering inclusion. 


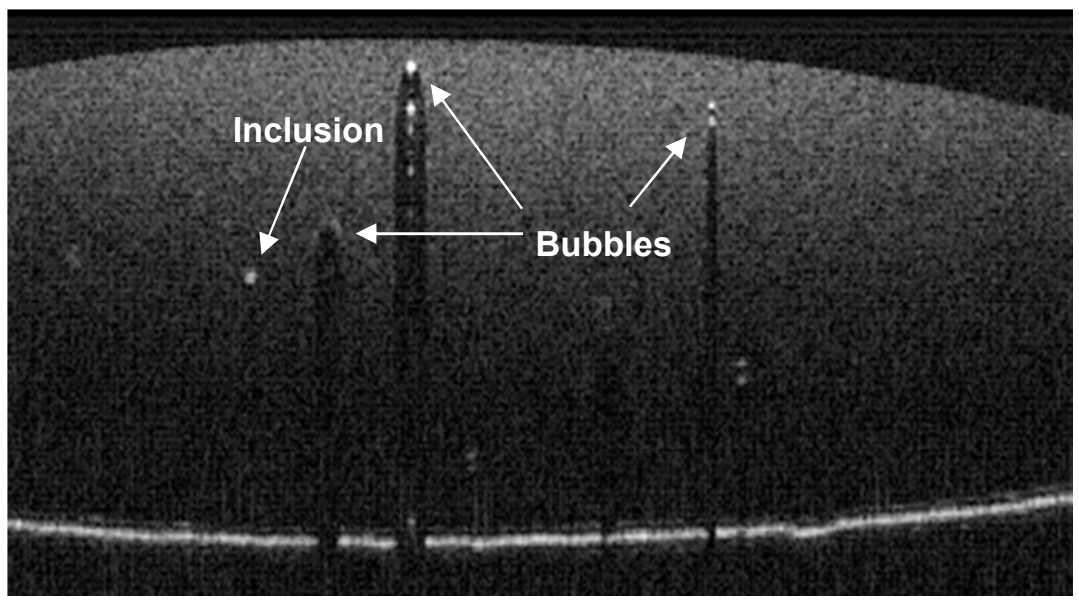

Figure 3. OCT image, containing a highly-scattering inclusion and bubbles, magnified to show more clearly the shadowing beneath bubbles. The top two bright spots on the shadow centre-line correspond to upper and lower bubble surfaces, while lower bright spots are artefacts arising from the strong specular reflections.

For correlation intervals longer than about two minutes, correlation values sometimes drop slightly in regions of the imaged slice, as can be seen in figure 2 for the 30 -minute correlation image. There are several possible reasons: polarisation changes in the optical fibres, causing a reduction in the amplitude of the interferometric fringes, or bulk sample movement, could both result in de-correlation, but identical changes would be expected at all points across the image. Air currents across the sample can cause position-sensitive de-correlation, and this is indeed observed when a strong puff of air is deliberately caused over the sample during acquisition. However, shielding of the sample did not eliminate the artefacts seen in the Araldite images; they were also more persistent than would be expected from the transient effects of air movements.

To further investigate de-correlation effects, a rigid sample (small piece of coral) was monitored at 15second intervals over several minutes, each image being correlated with an original reference image acquired at zero time. De-correlation artefacts typically emerge after the first two minutes or so, and tend to occur in similar positions over time-frames of a few minutes. Sometimes they recover slightly, but further de-correlation then occurs at other sites, with a general trend to poorer correlation over time. It appears likely that the main cause is non-homogeneous, micrometre-scale thermally-induced deformation of probe head components, mounting plates, the sample itself or all three, resulting in 
gradual alteration of the speckle within the images. Correlation intervals should preferably be kept below about two minutes to minimise such artefacts.

Possible vibration effects should be considered. Image acquisition takes several tens of milliseconds, therefore vibration of appreciable amplitude above about $20 \mathrm{~Hz}$ causes periodic banding to appear in OCT images, which cannot readily be eliminated in software. Lower frequency vibration will appear as reversible, correlation interval-dependent image de-correlation. This study was performed on a well-isolated optical table, and vibration artefacts were not seen.

In some circumstances, non-homogeneous correlation changes will arise as a genuine feature, caused by inhomogeneous movement within the cure volume. If cross-linking occurs at different rates in differently-mixed regions of adhesive, stresses will be set up which could be affected by temperature changes or, in the semi-solid state, released by a movement having aspects both of bulk motion and flow, to differing degrees in different regions of the mixture, in a manner analogous to a small 'earthquake' or slippage between neighbouring regions. However, it is unlikely that such effects would be observed after more than 18 hours of cure, and mechanical drift is a more likely explanation in this situation. Semi-solid flow effects, if indeed they occur in epoxy adhesive mixtures, should be minimised by thorough mixing of the two epoxy components.

\section{3 'Araldite Rapid' epoxy: poorly-mixed sample}

In a second experiment, another sample of 'Araldite Rapid' epoxy was mixed, as directed on the packaging, but was stirred for only a few seconds so that mixing was poor relative to that in the previous experiment. As before, a thin line of the mixture, about a millimetre thick, was deposited on the metal substrate plate.

The appearance of the raw OCT images for this mixture, shown in figure 4, was clearly very inhomogeneous. The resin component of this epoxy is semi-opaque, while the hardener is optically clear, so we presume that the regions of high scattering correspond to resin-rich areas of the mix, while regions of low scattering indicate a higher proportion of hardener. The fine detail of structural variations is less apparent in the correlation images, but some interesting temporal effects are 


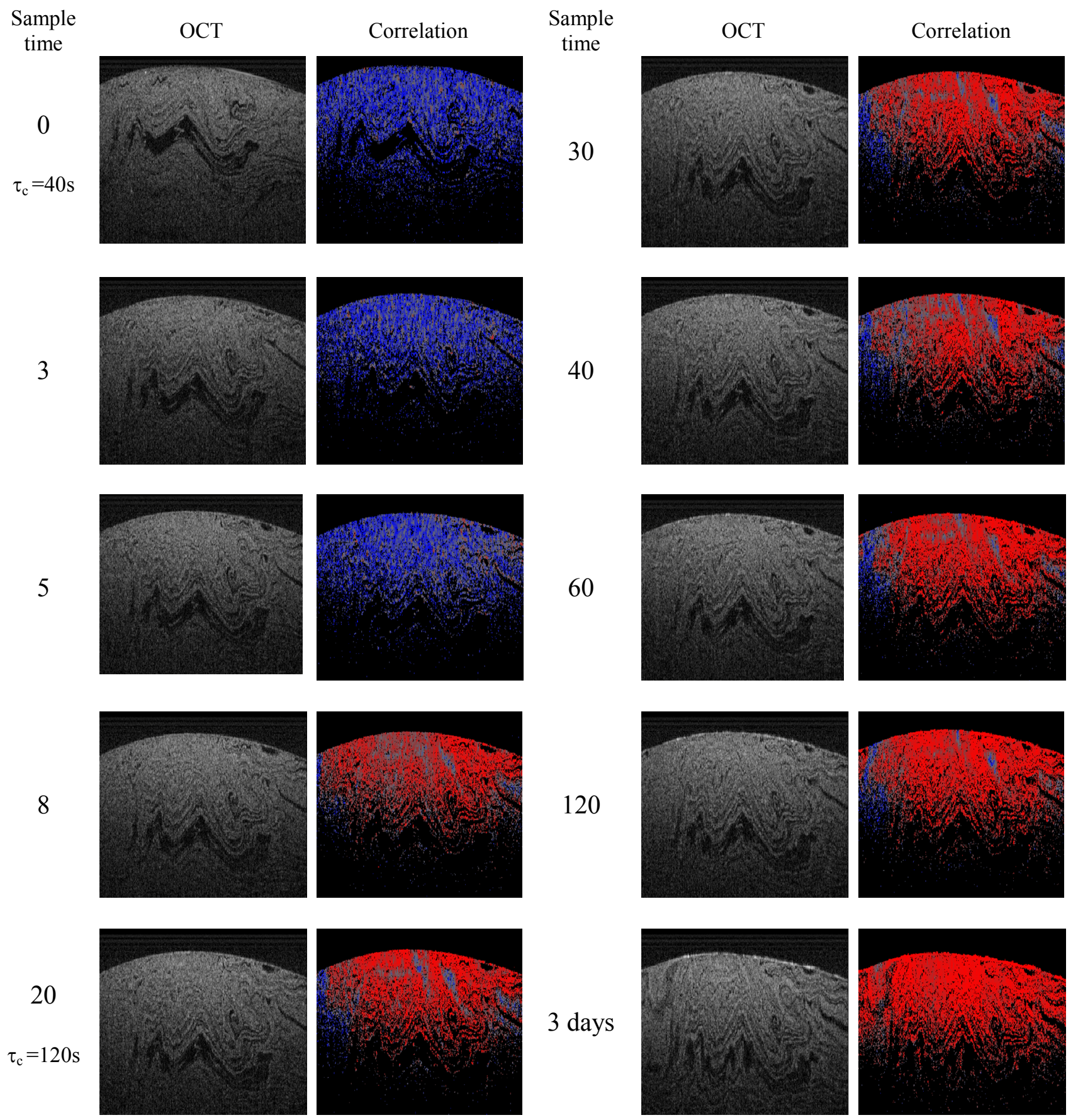

Figure 4. OCT (left) and correlation (right) images for a poorly-mixed 'Araldite Rapid' epoxy sample. Image dimensions are $3 \mathrm{~mm}$ laterally by $1.6 \mathrm{~mm}$ deep. The sampling time given for each image pair is in minutes. The correlation interval is $40 \mathrm{~s}$ for the first four image pairs shown, and $120 \mathrm{~s}$ thereafter. Colour scale is as for figure 1 .

observed as cure progresses in this sample. Appropriate correlation intervals and sampling intervals for different stages of cure were selected as before, and are listed in table 1. For the first five minutes, very little change was seen, using these parameters, but a large and rapid increase in average viscosity 
then occurred over the following four to five minutes. This is consistent with the ten-minute cure time quoted for the mixed adhesive. The cure process was followed as it progressed more slowly over the following two hours, increasing the correlation and sampling intervals appropriately as the speed of cure slowed from its initial rate.

It is interesting to note the persistence of some regions of low correlation in this poorly-mixed sample, over very long time periods. On the far left of each correlation image in figure 4, a large reservoir of low-viscosity fluid is present (dark grey, or blue online), which exhibits a $\tau_{\mathrm{c}}$ of only about 30 seconds, even after 80 minutes setting time, and is still not fully cured in the images acquired after 3 days. It is possible to gauge the degree of shrinkage during cure, from the rightwards lateral migration of this zone between image acquisitions. Interestingly, there is much less evidence of a problem in this uncured region from the original OCT images, presumably because it is comprised mainly of unmixed resin, and therefore scattering is similar to that in the better-mixed areas. Other, smaller, islands of low-viscosity fluid can also be identified in correlation images acquired up to two hours after mixing, although these have disappeared in the 3-day image. This provides a powerful tool for the development of mixing protocols, and allows linking of OCT-observable cure defects to possible mechanical weaknesses in cured samples of adhesive; sizeable regions that contain either voids (identifiable from the raw OCT images), or regions of poor correlation, are indicators of places where cracks or de-laminations might be initiated or spread with rapidity through the cured product. Scattering particles added to the hardener, would improve the identification of uncured regions of hardener from the correlation images, as well as those of resin, in supposedly fully-cured samples.

\subsection{General discussion}

The benefit of a localised measurement of cure will depend on the particular application. Often, it is sufficient simply to know when cure is complete, in which case the spatial information obtained from an OCT measurement is unnecessary. However, there are situations in which spatially-resolved information is valuable. For example, when bonding fragile materials, the stresses imparted to the pieces to be joined will change significantly depending on whether the adhesive cures from the 
outside in, from the inside out, or homogeneously, and on the relative rate at which cure progresses in each region. When two components must be mixed, as in the Araldite Rapid epoxy discussed above, the ability to see structure inside the curing adhesive will allow possible weak points of poor mixing to be identified, and to be compared with fracture behaviour of the adhesive under stress.

In this paper we have studied relatively thick layers of adhesive, whereas in practice much smaller quantities would often be used. However, much useful information about the general behaviour of the materials can be learned, that will apply also to thinner layers. Although the spatial resolution in the raw OCT images is only $7-8 \mu \mathrm{m}$, the image correlation process increases this significantly due to averaging over the area of the kernel. It is still possible to obtain structural images and average viscosity information in more realistic layer thicknesses, down to about $30-50 \mu \mathrm{m}$, but the relative timescales in different regions of the mixture will not be resolved at this length scale. When the aim is to make measurements in-situ, it is obviously necessary for the sample to have a reasonable degree of optical clarity at the wavelength used. A satisfactory depth of image will not be obtained in materials that are either opaque or very strongly scattering. Conversely, imaging will also be poor if the material is optically clear and no scattering centres are present, although this problem could be alleviated, in some cases, by the deliberate introduction of scattering particles. Optical access to the region of interest is essential so, where the adhesive is being used to bond opaque materials, it will only be possible to perform in-situ monitoring for a real application if a transparent analogue of the parts to be bonded can be constructed.

In addition to the spatially- and temporally-resolved information, obtained as discussed above, the depth-averaged group refractive index $\mathrm{n}_{\mathrm{g}}$ of the adhesive can also be obtained, as a function of time and lateral position, provided that the optical penetration is sufficient for the substrate surface to seen at all lateral positions, and that an all-air path to the substrate is included in the image. Extraction of the group refractive index is a well-known OCT technique [24] that could, in this application, provide additional useful information about the cure process. The measurement is achieved by taking the ratio of the optical distance $n_{\mathrm{g}} l$, between the upper and lower surfaces of the bead, as measured by the OCT 
instrument, to the physical bead thickness $l$ at the same lateral position, inferred from the imaged distances in air to both the substrate plate and to the upper surface of the bead.

\section{Conclusions}

Both conventional OCT imaging and a recently-reported OCT correlation technique have been applied to the imaging of adhesives, to permit spatially-resolved cure-monitoring of a PVA paper glue and a two-part epoxy resin. Information on the relative viscosity in different regions is obtained, via a local correlation time constant, with a spatial resolution of a few tens of micrometres and a temporal resolution of a few seconds. Indications of phase changes have been observed in the emulsion-based glue during drying, and mix-dependent inhomogeneity of cure in an epoxy resin sample. Information about locations of imperfections within the materials can also be obtained, with a spatial resolution of about 7-8 $\mu \mathrm{m}$, from the raw OCT images. We believe this is the first time that such high-resolution, spatially-resolved information has been obtained in adhesive cure-monitoring.

\section{Acknowledgements}

We acknowledge the support of a Platform Grant EP/H02252X/1 from the Engineering and Physical Sciences Research Council (EPSRC), UK.

\section{References}

[1] D. R. Mulligan, Cure monitoring for composites and adhesives, RAPRA review reports 14, 8 2003.

[2] M. J. Lodeiro and D. R. Mulligan, Cure monitoring techniques for polymer composites, adhesives and coatings, National Physical Laboratory (NPL) Measurement good practice guide no.75, February 2005.

[3] C. Meola and G. M. Carlomagno, Application of infrared thermography to adhesion science, J. Adhes. Sci. Technol. 20 (2006) 589-632.

[4] G. M. Maistros and I. K. Partridge, Monitoring autoclave cure in commercial carbon fibre/epoxy composites, Composites Part B: Eng. 29 (1998) 245-250.

[5] D. Chadwick, Cure monitoring, US Patent 6,675,112 B1, Jan 62004. 
[6] D. D. Shepard and K. R. Smith, Ultrasonic cure monitoring of advanced composites, Sens. Rev. 19 (1999) 187-194.

[7] H. Zhang and J. Mijovic, Using two-dimensional dielectric relaxation spectroscopy to study the effect of water on the dynamics of epoxy-amine networks, Macromolecules 37 (2004) 5844-5846. [8] F. W. Wang, R. E. Lowry and B. M. Fanconi, Novel fluorescence method for cure monitoring of epoxy resins, Polymer 27 (1986) 1529-1532.

[9] J. Mijovic and S. Andjelic, Study of the mechanism and rate of bismaleimide cure by remote insitu real time fibre-optic near-infrared spectroscopy, Macromolecules 29 (1996) 239-246.

[10] J. R. Dunphy, G. F. Meltz, P. Lamm and W. W. Morey, Multi-function, distributed optical fiber sensor for composite cure and response monitoring, Fiber Optic Smart Structures and Skins III, San Jose (1990) 116-118.

[11] E. Chehura, M. Kazilas, S. W. James, I. K. Partridge and R. P. Tatam, Strain Development in Curing Epoxy Resin and Glass Fiber/Epoxy Composites Monitored by Fibre Bragg Grating Sensors in Birefringent Optical Fibre, Smart Materials and Structures 14 (2005) 354-362.

[12] C. Fernandez-Valdivielso, I. R. Matias and F. J. Arregui, Simultaneous measurement of strain and temperature using a fiber Bragg grating and a thermochromic material, Sens. and Actuators A 101 (2002) 107-116.

[13] S. J. Buggy, E. Chehura, S. W. James and R. P. Tatam, Optical fibre grating refractometers for resin cure monitoring, J. Opt. A: Pure Appl. Opt. 9 (2007) S60-S65.

[14] A. F. Fercher, C. Hitzenberger and M. Juchen, Measurement of intraocular optical distances using partially coherent laser light, J. Mod. Opt. 38 (1991) 1327-1333.

[15] A. F Fercher, W. Drexler, C. K. Hitzenberger and T. Lasser, Optical coherence tomography principles and applications, Rep. Prog. Phys. 66 (2003) 239-303.

[16] E. A. Swanson, J. A. Izatt, M. R. Hee, D. Huang, C. P. Lin, J. S. Schuman and J. G. Fujimoto, Invivo retinal imaging by optical coherence tomography, Opt. Lett. 18 (1993) 1864-1866. 
[17] J. Enfield, E. Jonathan and M. Leahy, In vivo imaging of the microcirculation of the volar forearm using correlation mapping optical coherence tomography (cmOCT), Biomed. Opt. Exp. 2 (2011) 1184-1193.

[18] J. G. Fujimoto, Optical coherence tomography for ultrahigh resolution in-vivo imaging, Nat. Biotechnol. 21 (2003) 1361-1367.

[19] J. D. Briers and A. F. Fercher, Retinal blood-flow visualization by means of laser speckle photography, Investig. Ophthalmol. Vis. Sci. 22 (1982) 255-259.

[20] P. Debye, Molecular weight determination by light scattering, J. Phys. Chem. 51 (1947) 18-32.

[21] A. Einstein, Investigations on the theory of Brownian movement, ed. R Furth, translated by A D Cowper, Einstein, collected papers, Dover publ., 1956.

[22] F. Reif, Fundamentals of Statistical and Thermal Physics, Ch. 12, 484, McGraw-Hill, Inc., Tokyo, Japan, 1965.

[23] M. A. Choma, M. V. Sarunic, C. H. Yang and J. A. Izatt, Sensitivity advantage of swept source and Fourier domain optical coherence tomography, Opt. Exp. 11 (2003) 2183-2189.

[24] S. Lawman, H. Liang, High precision dynamic multi-interface profilometry with optical coherence tomography, Appl. Opt. 50 (2011) 6039-6048. 